\title{
Electronically tunable aperiodic distributed feedback terahertz lasers
}

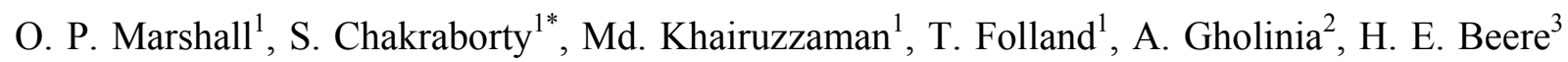
and D. A. Ritchie ${ }^{3}$

${ }^{1}$ School of Electrical and Electronic Engineering, University of Manchester, Manchester M13 9PL, UK.

${ }^{2}$ School of Materials, University of Manchester, Manchester M1 7HS, UK.

${ }^{3}$ Cavendish Laboratory, Department of Physics, University of Cambridge, Cambridge CB3 0HE, UK.

*e-mail: s.chakraborty@manchester.ac.uk

\section{ABSTRACT}

Focussed ion beam milling can be used to introduce aperiodic distributed feedback (ADFB) gratings into fully packaged, operational terahertz quantum cascade lasers to achieve electronically controlled, discretely tunable laser emission. These aperiodic gratings designed using computer-generated hologram techniques - consist of multiple slits in the surface plasmon waveguide, distributed along the length of the laser cavity. Tuning behaviour and output power in ADFB lasers operating around 2.9 THz are investigated with a variety of slit dimensions and grating scales. Mode selectivity and grating losses are found to be strongly dependent on milling depth into the upper waveguide layers, dramatically increasing as the metallic layers are penetrated, then rising more slowly with deeper milling into the laser active region. Grating scale and placement along the laser cavity length are also shown to influence mode selection. 


\section{INTRODUCTION}

The properties of terahertz ( $\mathrm{THz}$ ) radiation, commonly defined as $300 \mathrm{GHz}$ to $10 \mathrm{THz}(1 \mathrm{~mm}$ to $30 \mu \mathrm{m})$, make it particularly appealing for a number of applications. Its penetration in many common materials, including plastics, paper and clothing, is combined with a wealth of spectral information; many substances possess strong absorption features at $\mathrm{THz}$ frequencies. Furthermore, low photon energies mean that $\mathrm{THz}$ radiation is non-ionising and therefore inherently safer and less destructive than x-ray systems. As a consequence there exists a growing interest in $\mathrm{THz}$ applications in fields as diverse as security ${ }^{1}$, spectroscopy $^{2}$ and medicine ${ }^{3}$, amongst others. Unfortunately, many of today's competing $\mathrm{THz}$ source technologies suffer drawbacks, including low emission powers, and high system costs and complexity ${ }^{4}$. Since their first demonstration by Köhler et al in $2002^{5}$, THz quantum cascade lasers (QCLs) have promised to be the technological answer to the question of a compact, electrically driven, powerful and coherent $\mathrm{THz}$ source. Nevertheless, despite a decade of continuous improvements in performance, the market potential of THz QCLs has not yet been fulfilled. Two obstacles to the widespread adoption of $\mathrm{THz}$ QCLs have been their cryogenic operating temperatures and a lack of frequency-selective tunability in standard devices. Ongoing improvements in active region design and packaging continue to drive up the operating temperature ceiling. For example, the recent demonstration of THz QCL operation at $200 \mathrm{~K}$ is well within reach of compact, cryogenfree cooling systems ${ }^{6}$. On the other hand, the problem of electrically controlled, single-mode tuning has proven more difficult to solve. Active region (AR) modification has opened up the possibility of coarse global frequency tuning ${ }^{7-11}$, but does not intrinsically provide precise frequency discrimination. Various distributed feedback techniques have been used for precise frequency selection in single- or dual-mode emitting $\mathrm{THz}$ QCLs ${ }^{12-15}$, but do not produce 
significant electronic tuning. Until recently, only mechanically adjustable waveguides or external waveguide arrangements had achieved mode selection and tuning effects simultaneously ${ }^{16,17}$, giving tunable yet single-colour emission from a solitary THz QCL. Another approach has since been demonstrated by the authors, with discrete electronic tuning of THz QCL emission across a range of user-defined frequencies using an aperiodic distributed feedback (ADFB) grating ${ }^{18}$. The grating contains a multitude of quarter-wave phase-shifts with precise locations and sizes, set in such a way that they operate collectively to provide a well-defined set of spectral reflectivity resonances. More specifically, the ADFB grating is a longitudinal computer-generated hologram (CGH), which digitally encodes the Fourier transform (FT) of a target spectral response function ${ }^{19}$. In order to achieve switchable, single mode THz QCL emission, this response must contain multiple resonances within the gain bandwidth of the laser. The ADFB gratings were introduced to pre-fabricated, fully packaged THz QCLs displaying highly multi-moded emission and electrical tuning of the gain centre frequency. Focussed ion beam (FIB) milling was used to create multiple sub- $\mu \mathrm{m}$ slits in the upper layers of the plasmon waveguide of the QCLs ${ }^{20}$, following the pattern of the ADFB grating design ${ }^{18}$. The intention is that each grating reflection resonance then causes lasing on a single dominant frequency as the underlying QCL gain peak is shifted with driving current. The FIB post-processing approach has advantages over direct incorporation of the ADFB grating at the initial QCL fabrication stages. It enables tailoring of the ADFB spectral response to individual lasers. Also, the fact that devices may be characterized before and after FIB milling, allows the affect of the ADFB grating on the lasing spectra and output power to be clearly discerned. Here we investigate the performance characteristics of ADFB-controlled THz QCLs as the grating properties, such as milling depth and spectral response, are varied. 


\section{WAVEGUIDING AND APERIODIC GRATING DESIGN}

In this work, all lasers employed semi-insulating surface plasmon (SI-SP) waveguides. Device fabrication is described in detail later, but a typical packaged QCL schematic and its waveguide layer structure are given in Figs. 1a and $1 \mathrm{~b}$ respectively. Figure 1c shows a simulated crosssectional fundamental optical mode intensity profile for an unperturbed, $150 \mu \mathrm{m}$ wide SI-SP ridge. The plasmonic nature of the SI-SP waveguide makes this mode profile highly sensitive to the properties of the upper metallic layers. Figure 1d shows the modified mode profile after removal of the metal overlayers in the simulation. Both components of the complex effective refractive index $\left(n_{\text {eff }}\right)$ are significantly altered. Compared to figure $1 \mathrm{c}$, the change in $n_{\text {eff }}$ is calculated as 0.339 - i0.21, giving a complex refractive index contrast of amplitude $|\Delta n|=0.399$. Furthermore, the cross-sectional mode shape changed such that the overlap with the AR $(\Gamma)$ is reduced from 23 to $11 \%$. These simulations illustrate the possibility of introducing a considerable spatial variation in the modal refractive index simply by patterning these metal layers (e.g. by FIB milling), without the need to remove any of the underlying AR or substrate material. In fact, when patterning SI-SP laser waveguides in this way, care must be taken not to reduce the modal $\Gamma$ too far. This will serve to reduce the net modal gain and therefore hinder laser action. For this reason, ADFB grating slits are kept very short (usually $<1 \mu \mathrm{m}$ ), giving duty cycles in excess of $90 \%$. Such small features might usually be achieved using standard lithographic techniques, but these are precluded by device packaging. This drawback is however outweighed by the enormous advantage of knowing the specific spectral behaviour of each QCL prior to introducing the ADFB grating. Small variations in AR thickness and device processing can create differences between individual devices. Knowledge of the gain behaviour is crucial for the correct design of the ADFB grating, whereas individual device characterization enables 
precise spectral placement of the ADFB response for each QCL. Hence, as QCLs are packaged and characterized prior to grating introduction, an alternative, direct method of pattern transfer was required. Focussed ion beam (FIB) milling was identified as the only viable approach, allowing precisely controlled removal of material without the need for pre-patterning the device. Techniques using photons rather than ions as the tool of choice, (e.g. laser ablation), were ruled out due to the required feature sizes; sub-micron features would require prohibitively short photon wavelengths.

Three key parameters influence the final ADFB design: the required number of pixels for the hologram, the minimum slit separation $\Lambda$ and $|\Delta n|$. For a hologram containing $2 N$ pixels, the total grating length $L$ is equal to $N \Lambda$. Changing $\Lambda$ serves to uniformly stretch or compress the grating, with a resulting shift in its central (Nyquist) frequency $f_{\mathrm{N}}=\mathrm{c} / 2 n_{\mathrm{eff}} \Lambda$, where $\mathrm{c}$ is the speed of light in vacuum. On the other hand, the minimum resonance spacing $\Delta f$ is dictated by $L$, as $\Delta f \approx \mathrm{c} /\left(2 n_{\mathrm{eff}} L\right)$. Hence, in the target spectral response of an ADFB, the normalized resonance spacing, expressed as $\Delta f / f_{\mathrm{N}}=1 / N$, is purely controlled by the hologram pixel number. For an ADFB grating-modified QCL to operate as intended, the correct $f_{\mathrm{N}}$ and $\Delta f$ must be achieved. Assuming $n_{\text {eff }} \approx 3.68$, a value of $\Lambda \approx 14.2 \mu \mathrm{m}$ is required such that $f_{\mathrm{N}}$ lies close to $2.9 \mathrm{THz}$. Furthermore, to ensure highly delineated resonances every other resolvable frequency point was selected. Hence, in order to achieve multiple resonances within the gain bandwidth of the $\mathrm{THz}$ QCLs, $\Delta f=30 \mathrm{GHz}$ was chosen, resulting in $L \approx 3 \mathrm{~mm}(N=200)$. Such a long grating has implications for the target spectral response. Modelling results suggest that for optimal discrete mode tuning, the reflectivity resonances generated by the ADFB grating must be limited in strength to match the $\sim 30 \%$ cleaved laser facet reflectivity ${ }^{18,21}$. If resonances are weaker they will not provide sufficient spectral filtering, stronger and they hinder frequency migration (i.e. 
switching) under varying laser driving conditions. Resonance strengths are controlled by the product of two terms, namely $|\Delta n|$ and the spectral components of the FT of the binary grating distribution $^{19}$. Ideally, in order to achieve $30 \%$ reflectivity in a long structure, $|\Delta n|$ must be small. In this work a value of $|\Delta n|=0.1$ was chosen and the FT components tailored accordingly. For the surface plasmon waveguide system, fine control of $|\Delta n|$ with milling depth is difficult due to the large and abrupt response of $n_{\text {eff }}$ to the removal of waveguide layers (Fig. 1d), however $|\Delta n|$ is effectively be reduced by the high ADFB grating duty cycles. It must be noted that once the ADFB design is fixed, any rise in $|\Delta n|$ will increase the grating reflectivity over its entire frequency range, and vice versa.

\section{FABRICATION AND CHARACTERIZATION}

All THz QCLs were fabricated from a single molecular beam epitaxially (MBE) grown GaAs/ $/ \mathrm{Al}_{0.15} \mathrm{Ga}_{0.85} \mathrm{As}$ wafer and employed $180 \mu \mathrm{m}$ wide by $\sim 6 \mathrm{~mm}$ long SI-SP ridge waveguides ${ }^{5}$. The active region produced emission around $2.9 \mathrm{THz}$, with a trend towards higher frequency lasing modes at higher driving currents/biases ${ }^{9}$. After ridge definition by wet chemical etching, ohmic contacts and metal overlayers were deposited by thermal evaporation. Metal thicknesses were carefully calibrated and controlled. Laser cavities were defined by mechanical cleaving, devices were indium soldered to copper heatsinks and finally wire bonded for electrical contact (Fig. 1a).

Device characterization was performed in pulsed operation $(1 \%$ duty cycle, $1 \mu$ s pulse length) at liquid helium temperatures in a Janis ST-100 continuous flow cryostat. Output powers were recorded using a calibrated, large area thermopile detector placed inside the cryostat, 
directly in front of the QCL. High resolution $\left(0.075 \mathrm{~cm}^{-1}\right)$ emission spectra were recorded using a nitrogen-purged Bruker Vertex 80 Fourier transform infrared (FTIR) spectrometer.

After initial characterization, ADFB gratings were introduced to the THz QCLs by FIB milling. An FEI Nova Nanolab 600 FIB system with a high-precision piezo-motor driven sample stage was used in this work. A $30 \mathrm{kV}$ Ga ion beam at $1 \mathrm{nA}$ beam current, producing a nominal spot size of $50 \mathrm{~nm}$, was chosen as a compromise between achievable milling resolution and writing time of the ADFB grating. Under these conditions the milling time was approximately 40 minutes. Unfortunately, at the minimum system magnification the writable field width was limited to $800 \mu \mathrm{m}$, and hence the $\sim 3 \mathrm{~mm}$ long grating had to be written in multiple stitched sections. Initial milling trials revealed some pattern distortion and an increase in beam spot size towards the outlying regions of the workable field. For this reason a $630 \mu \mathrm{m}$ section size was chosen, giving five sections with $30 \mu \mathrm{m}$ overlaps between neighbouring sections. As evidence that the pattern distortion was minimal over each section, measurements with the high-precision stage and scanning electron microscope revealed section lengths of $630 \pm 2 \mu \mathrm{m}$, equivalent to an error of $\pm 0.3 \%$. Assuming this error is distributed evenly along the ADFB structure, it would introduce an error on $f_{\mathrm{N}}$ of $\pm 0.003 f_{\mathrm{N}}$. For a typical $f_{\mathrm{N}}$ of $2.9 \mathrm{THz}$ this equates to a real frequency error of $\pm 8.7 \mathrm{GHz}$ and a $\Delta f$ error of $\pm 87 \mathrm{MHz}$. Sample charging effects were minimised by electrically shorting all parts of the packaged QCL to the sample holder. In fact, the existing bond wires and packaging aided electrical grounding by ensuring the presence of a conductive path away from the laser ridge surface. After sample mounting and evacuation of the FIB chamber, the system was left for at least 30 minutes to ensure thermal stability. Finally, just prior to the final pattern milling, an inactive area of the device, away from the laser ridge, was FIB milled using the ADFB pattern. In part, this milling was a precaution against beam drift in the 
first ADFB section caused by any residual device charging effects from imperfect grounding, or by any initial sample temperature rise at the onset of milling. A scanning electron microscope (SEM) image of a packaged and milled QCL ridge is shown in Fig. 2a. The grating is placed approximately equidistant from each cleaved facet. Evidence of milling into the uppermost electrical contact (an optically inactive area) is also visible. The ADFB grating consists of 177 slits, perpendicular to the laser cavity and at precisely defined positions, with a number of $\Lambda / 2$ phase shifts dispersed throughout the structure. For clarity, a symbolic representation of the grating design is given in Fig. 2b. Upon closer inspection, the intended slit pattern is clearly discernable in the grating region of the QCL ridge (Fig. 2c). Slit widths were deliberately kept narrower $(100 \mu \mathrm{m})$ than the QCL ridge $(180 \mu \mathrm{m})$ in order to maintain electrical contact to the entire ridge length via the upper metal layers, as ridges were only wire-bonded at either end. Ideally, slits should be identical to achieve the designed spectral response. Large variations in slit dimensions may lead to undesirable changes to the final reflectivity response function. Nevertheless, the fact that the ADFB is essentially a digital hologram makes these structures fairly robust against individual pixel imperfections. Figure $2 \mathrm{~d}$ shows a short section of grating structure, revealing multiple identical slits and one of the numerous phase shifts. As noted, the grating has a duty cycle in excess of $90 \%$ due to the short slit lengths. Consequently, $\Gamma$ was maintained at an acceptable level and the additional waveguide losses introduced along with $|\Delta n|$ were minimised. Detail of a single slit, with a length $l \approx 500 \mathrm{~nm}$, is shown in Fig. 2e. Slit depths (z) were varied from device to device, but were always shallow relative to the $\sim 12 \mu \mathrm{m}$ ridge height. In the absence of an end point detection mechanism, $z$ was controlled by the milling duration. It must be noted that there is a variation of $z$ within each slit, with what appears to be a series of holes. This is attributed to the differing milling rates of the constituent layers within the 
QCL waveguide. Mechanically resistive titanium and palladium layers sit directly above GaAs. There exist small non-uniformities in the thickness of these thermally evaporated metals. The roughness of the PdGe/GaAs interface in discernable in Fig. 2f. This spatial variation leads to penetration by the ion beam at slightly different times. Regions punctured first then appear as holes due to the higher milling rate of the underlying semiconductor. In practice only the average milling depth within the slit was relevant to device performance as the internal hole and filament texture is highly sub-wavelength in the $\mathrm{THz}$ regime. Figure $2 \mathrm{f}$ shows a cross section through a single slit of moderate depth, penetrating the metallic layers and the $80 \mathrm{~nm}$ thick highly doped GaAs layer below. During FIB milling, small additional patterns were milled alongside the QCL ridge with each of the five grating sections. The patterns from neighbouring sections were designed to align as a means to inspect the section stitching accuracy after milling. The pattern is illustrated in Fig. $2 \mathrm{~g}$ and its typical milled appearance shown in Fig. 2h. In all cases, stitching was found to be very precise, with stitching errors of $\leq 1 \mu \mathrm{m}$ between sections in the direction of the laser axis. Note that the dimensional tolerances outlined above, requiring simultaneous precision over length scales ranging from a few millimetres $(L)$ down to hundreds of nanometres ( $l$ and $z$ ), placed strict constraints on the FIB milling. Despite the inherent flexibility of the FIB process, these tolerances presented multiple challenges to device fabrication. Precise placement and milling of sub- $\mu \mathrm{m}$ scale features, into a target of varied material layer structure, over a length scale of millimetres, falls outside the scope of standard FIB-milling practices and was non-trivial to produce.

\section{RESULTS}

Figure 3 shows selected emission spectra from six THz QCLs (devices $A$ to $F$ ) both before (dashed lines) and after (solid lines) ADFB grating introduction. The target multi-band ADFB 
reflectivity response and its spectral placement relative to the laser emission are also given for each device. From their spectral behaviour, the ADFB lasers may be grouped into three categories. Devices $A$ and $B$ show minimal grating influence, with spectra resembling the initial Fabry-Perot (FP) emission; multiple lasing modes separated by the longitudinal cavity mode spacing of $\sim 6.6 \mathrm{GHz}$. Devices $C$ and $D$ behave differently, lasing instead on switchable, ADFB controlled modes, with a frequency spacing closely matching that of the ADFB resonances $(\sim 30 \mathrm{GHz})$. Finally, devices $E$ and $F$ operate on only two or three frequencies each across the entire span of the QCL gain. These three distinct spectral behaviours are attributed to differing regimes of ADFB influence: weak, moderate and strong respectively. When too weak, the grating feedback is small compared to the facet reflectivity, leading to an FP-like spectral power distribution. Too strong and the system is entirely ADFB controlled, with only the most highly favoured mode solutions achieving lasing. As explained in detail in reference 21, the target behaviour of discrete tuning between ADFB modes only occurs with a moderate grating influence. As the same basic ADFB design was used in each case, the differences between the three categories of laser in Fig. 3 are primarily due to variation in $|\Delta n|$; higher index contrasts producing a larger ADFB influence over the QCL.

Figure 4a shows the electrical and output power characteristics for one QCL from each spectral category: devices $A, C$ and $E$. Prior to FIB milling each device showed almost identical electrical performance and lasing threshold current density $J_{\text {th }}$ (just below $100 \mathrm{~A} \mathrm{~cm}^{-2}$ ). Peak output powers were also comparable, ranging from 24 to $28 \mathrm{~mW}$. After milling differences were once again observed between the weak (device $A$ ), moderate $(C)$ and strong $(E)$ grating influences. The new threshold current densities $J_{\text {th }}{ }^{\text {FIB }}$ are higher than the original $J_{\text {th }}$ values, though by varying amounts for each laser, increasing by $1 \%, 10 \%$ and $25 \%$ for devices $A, C$ and 
$E$ respectively. Only small changes are observed in the slope efficiencies and peak power driving currents. Consequently, the peak output powers for each laser are reduced by varying amounts, primarily dependent on the shift in $J_{\text {th }}$. The top illustration Fig. $4 \mathrm{~b}$ shows the structure and thickness of the upper layers of the SI-SP waveguide. Figure $4 \mathrm{~b}$ also contains representative SEM images of the slits in each device, which enable estimation of FIB milling depths. A direct measurement of slit depth would require cross-sectional analysis of multiple slits in each device at multiple locations. This non-reversible process was avoided as it would destroy (or severely damage) devices. Instead, slit depths were estimated by comparing the SEM images of Fig. $4 \mathrm{~b}$ to prior devices in which cross-sectional depth measurements were performed (e.g. Fig. 2f). Illustrations of the estimated (average) cross-sectional milling profiles for each group of devices are also presented in Fig. 4b. The estimated milling depth was then simplified to a single value by considering only the depth along the centre line of each slit. Various QCL performance characteristics may then be plotted against this estimated milling depth. For example, Fig. 4c shows the number of lasing modes (above a $10 \mathrm{~dB}$ cut-off level for individual normalised lasing spectra) across the entire operational current ranges of eight ADFB-modified QCLs. Due to the surface-plasmon nature of the layered SI-SP waveguides the relationship between milling depth and mode number is not linear. Little change in mode number (compared to the unperturbed FP spectra) is seen until the PdGe metal layers are penetrated (i.e. devices $C$ to $H$ ). Then, following a sharp drop in mode number, there are minimal further changes after the $n^{+}$-GaAs layer is penetrated. In Fig. 4c, the relationship between mode number and milling depth is approximated with an exponential trend line. A similar analysis can be performed to quantify the spectral purity of the ADFB QCL emission. After FIB milling, each QCL lases on a number of spectrally dominant modes. Taking the best achievable side-mode suppression ration (SMSR) for each 
dominant mode, then an average of these values, we can quantify the average SMSR of each device. These values are plotted against the estimated milling depth in Fig. 4d. Once again a clear (yet non-linear) relationship is observed and may be simply represented by an exponential trend line. The average SMSR values rise rapidly as the lower metallic waveguide layers (PdGe and $\mathrm{n}^{+}-\mathrm{GaAs}$ ) are removed, then more slowly with deeper milling into the QCL AR. The exponential trend line in Fig. 4d has the same exponent to that of Fig. 4c. Note that the average SMSR values are calculated from pulsed emission spectra with low duty cycles (1\%). By increasing the duty cycle it is possible to improve the SMSR further by minimising the effect of the non-zero rise time of the driving current pulses ${ }^{18}$. Finally, the additional optical losses associated with the FIB-milled ADFB grating are estimated from the changes in threshold current densities. Assuming facet reflectivities of $30 \%$, a median FP lasing wavelength $\lambda_{0}$ of $\sim 102 \mu \mathrm{m}$ and taking the simulated imaginary modal refractive index component $(k=0.007)$, the original FP waveguide and mirror losses are calculated as $\alpha_{\mathrm{w}} \approx 8.5 \mathrm{~cm}^{-1}$ and $\alpha_{\mathrm{m}} \approx 2 \mathrm{~cm}^{-1}$ respectively. With increasing milling depth the lasing threshold rises by up to $\sim 25 \%$, from which an additional grating loss $\alpha_{\mathrm{FIB}}$ is calculated for each laser, with values up to $\sim 2.6 \mathrm{~cm}^{-1}$ for the deepest milling. Calculated $\alpha_{\text {FIB }}$ values are plotted against milling depth in Fig. 4e. The exponents of the trend lines in Figs. 4c-e were chosen to be identical, yet still closely follow the behaviour of the mode number, SMSR and $\alpha_{\mathrm{FIB}}$ data, revealing the underlying link between these parameters and the milling depth. Although only a simple model, the exponential trends highlight that over $70 \%$ of the change in each of the above parameters is expected to occur with $z<400 \mathrm{~nm}$, i.e. a milling depth above the interface between the $\mathrm{n}^{+}-\mathrm{GaAs}$ and the AR. 
From the results presented in Figs. 3 and 4 it is clear that the correct choice of $z$ is crucial to achieving mode selection and post-FIB output powers in ADFB-modified THz QCLs. However, there are other grating parameters which may influence device performance. One such parameter is $\Lambda$. Figure 5a shows emission spectra from device $G$, in which the slit dimensions were similar to device $D$, but $\Lambda$ was chosen to detune $f_{\mathrm{N}}$ (vertical dotted line) from the gain peak. The post-FIB emission spectra contain five distinct ADFB controlled lasing modes, but the switching effect is less pronounced, and device $G$ has poor SMSR at most driving current densities. This illustrates the need for correct spectral positioning of the ADFB response, i.e. that it is placed at the designed position relative to the QCL gain ${ }^{18}$. On the other hand, the poor SMSR in device $G$ may be a down to the mode selection mechanism itself. Figure 5 b shows emission spectra from a final QCL (device $H$ ), with a moderate ADFB grating strength. Its milling depth and post-FIB behaviour resemble devices $C$ and $D$, with discrete tuning to higher frequency mode solutions with increasing driving current. However, at low driving current densities (just above $J_{\text {th }}$ ) lasing is observed at $2.97 \mathrm{THz}$, well away from the gain maxima located at approximately $2.88 \mathrm{THz}$. This effect is attributed to the mode selection and switching mechanisms previously proposed for ADFB QCLs ${ }^{21-23}$. Competition between the ADFB and the cleaved facet feedback introduces wide variability into the threshold requirements of the possible mode solutions in the FIB-modified laser. Furthermore, this variable mode competition landscape is sensitive to changes in the complex refractive index distribution along the QCL. Favourable mode solutions are changed simply by changing the driving current and therefore the gain within the laser. Final lasing modes tend to occur close to the peak spectral gain, and switching directions tend to follow any movement in the gain. However, because of the large variation in modal threshold requirements it is possible (though unlikely) for a solution far from 
the peak gain to dominate the emission spectra (device $H$ ) or for multiple modes to be selected simultaneously (device $G$ ). Discrete tuning may also occur in a direction opposite to the underlying peak gain movement. The phase relationship between the ADFB grating and the cleaved facets, as initially defined by the cavity length and grating placement, affects this mode selection. In this work, the relative ADFB-facet phase was not controlled during FIB milling. This can explain the precise mode selection variability from device to device. While the precise relationship between ADFB-facet phase and mode selection are difficult to calculate, a fixed initial phase might improve the precise spectral reproducibility between devices. In future device iterations, some degree of initial phase control may be realistically achievable. Utilizing the sub$\mu \mathrm{m}$ movement precision of the sample stage within the FIB chamber, it is possible to place the ADFB grating relative to the facets with high precision, much better than the $\mathrm{THz}$ radiation wavelength $(\sim 30 \mu \mathrm{m}$ in the QCL).

\section{CONCLUSION}

A non-standard FIB procedure was developed for milling mm-scale photonic structures, with sub- $\mu \mathrm{m}$-scale features, designed to operate at the long wavelengths associated with $\mathrm{THz}$ radiation. This enabled rapid iterative prototyping of ADFB-modified THz QCLs. The influence of various ADFB grating parameters on laser emission was investigated. Devices were found to be highly sensitive to the FIB milling depth, and potentially sensitive to both the physical scale and placement of the gratings within the laser cavities.

\section{ACKNOWLEDGEMENTS}

This work was supported by EPSRC First Grant EP/G064504/1 and partly supported by HMGCC. 


\section{References}

[1] J. F. Federici, B. Schulkin, F. Huang, D. Gary, R. Barat, F. Oliviera, and D. Zimdars, “THz imaging and sensing for security applications - explosives, weapons and drugs," Semicond. Sci. Technol. 20, S266-S280 (2005).

[2] D. M. Mittleman, R. H. Jacobsen, R. Neelamani, R. G. Baraniuk, and M. C. Nuss, "Gas sensing using terahertz time-domain spectroscopy," Appl. Phys. B 67, 379-390 (1998).

[3] E. Pickwell, and V. P. Wallace, "Biomedical applications of terahertz technology," J. Phys. D: Appl. Phys. 39, R301-R310 (2006).

[4] M. Tonouchi, “Cutting-edge terahertz technology," Nat. Photon. 1, 97-105 (2007).

[5] R. Köhler, A. Tredicucci, F. Beltram, H. E. Beere, E. H. Linfield, A. G. Davies, D. A. Ritchie, R. C. Iotti, and F. Rossi, “Terahertz semiconductor-heterostructure laser," Nature 417, 156-159 (2002).

[6] S. Fathololoumi, E. Dupont, C. W. I. Chan, Z. R. Wasilewski, S. R. Laframboise, D. Ban, A. Mátyás, C. Jirauschek, Q. Hu, and H. C. Liu, "Terahertz quantum cascade lasers operating up to $\sim 200 \mathrm{~K}$ with optimized oscillator strength and improved injection tunnelling," Opt. Express 20, 3866-3876 (2012).

[7] G. Scalari, C. Walther, J. Faist, H. Beere, and D. Ritchie, "Electrically switchable, two-color quantum cascade laser emitting at 1.39 and $2.3 \mathrm{THz}, "$ Appl. Phys. Lett. 88, 141102 (2006).

[8] Freeman J R, Marshall O P, Beere H E and Ritchie D A 2008 Electrically switchable emission in terahertz quantum cascade lasers Opt. Express 16 19830-19835

[9] J. R. Freeman, C. Worrall, V. Apostopoulos, J. Alton, H. Beere, and D. A. Ritchie, "Frequency Manipulation of $\mathrm{THz}$ Bound-to-Continuum Quantum-Cascade Lasers," Photon. Tech. Lett. 20, 303-305 (2008). 
[10] S. P. Khanna, M. Salih, P. Dean, A. G. Davies, and E. H. Linfield, "Electrically tunable terahertz quantum-cascade laser with a heterogeneous active region," Appl. Phys. Lett. 95, 181101 (2009).

[11] L. Lever, N. M. Hinchcliffe, S. P. Khanna, P. Dean, Z. Ikonić, C. A. Evans, A. G. Davies, P. Harrison, E. H. Linfield, and R. W. Kelsall, "Terahertz ambipolar dual-wavelength quantum cascade laser," Opt. Express 17, 19926-19932 (2009).

[12] L. Ajili, J. Faist, H. Beere, D. Ritchie, G. Davies, and E. Linfield, "Loss-coupled distributed feedback far-infrared quantum cascade lasers," Electron. Lett. 41, 419-421 (2005).

[13] L. Mahler, A. Tredicucci, R. Köhler, F. Beltram, H. E. Beere, E. H. Linfield, and D. A. Ritchie, "High-performance operation of single-mode terahertz quantum cascade lasers with metallic gratings," Appl. Phys. Lett. 87, 181101 (2005).

[14] B. S. Williams, S. Kumar, Q. Hu, and J. L. Reno, "Distributed-feedback terahertz quantumcascade lasers with laterally corrugated metal waveguides," Opt. Lett. 30, 2909-2911 (2005).

[15] L. Mahler, A. Tredicucci, F. Beltram, C. Walther, J. Faist, H. E. Beere, D. A. Ritchie, and D. S. Wiersma, "Quasi-periodic distributed feedback laser," Nat. Photon. 4, 165-169 (2010).

[16] J. Xu, J. M. Hensley, D. B. Fenner, M. G. Allen, F. Beltram, H. E. Beere, and D. A. Ritchie, “Tunable terahertz quantum cascade lasers with an external cavity," Appl. Phys. Lett. 91, 121104 (2007).

[17] Q. Qin, B. S. Williams, S. Kumar, J. L. Reno, and Q. Hu, "Tuning a terahertz wire laser," Nat. Photon. 3, 732-737 (2009). 
[18] S. Chakraborty, O. P. Marshall, M. Khairruzzaman, C.-W. Hsin, H. E. Beere, and D. A. Ritchie, "Longitudinal computer-generated holograms for digital frequency control in electronically tunable terahertz lasers," Appl. Phys. Lett. 101, 121103 (2012).

[19] S. Chakraborty, M. C. Parker, and R. J. Mears, "A Fourier (k-) space design approach for controllable photonic band and localization states in aperiodic lattices," Photon. Nanostr. Fund. Appl. 3, 139-147 (2005).

[20] S. Chakraborty, T. Chakraborty, S. P. Khanna, E. H. Linfield, A. G. Davies, J. Fowler, C. H. Worrall, H. E. Beere and D. A. Ritchie "Spectral engineering of terahertz quantum cascade lasers using focused ion beam etched photonic lattices," Electron. Lett. 42, 404-405 (2006).

[21] S. Chakraborty, O. P. Marshall, C.-W. Hsin, M. Khairuzzaman, H. E. Beere, and D. A. Ritchie, Opt. Express 20, B306-B314 (2012).

[22] S. Chakraborty, C.-W. Hsin, O. Marshall, M. Khairuzzaman, H. Beere, and D. Ritchie, "Mode switching using weak aperiodic DFB gratings within Fabry-Pérot lasers," in PECSX: 10th International Symposium on Photonic and Electromagnetic Crystal Structures, Santa Fe, USA, 2012.

[23] S. Chakraborty, C.-W. Hsin, O. Marshall, and M. Khairuzzaman, "Switchable Aperiodic Distributed Feedback Lasers: Time Domain Modelling and Experiment,” in Conference on Lasers and Electro-Optics, San Jose, USA, 2012. 
Figure 1. (a) Illustration of a packaged $6 \mathrm{~mm}$ long THz QCL. (b) Layer structure within the QCL. Simulated cross-sectional fundamental mode profiles within (c) a standard SI-SP waveguide at $2.85 \mathrm{THz}$ and (d) the same structure after the removal of the metal overlayers. Scale bars $50 \mu \mathrm{m}$. 
Figure 2. (a) Composite SEM image of an entire $6 \mathrm{~mm}$ long THz QCL ridge, showing electrical contacts, bond wires and ADFB region (dashed line). Scale bar $500 \mu \mathrm{m}$. (b) ADFB grating design. Vertical lines represent milled slits with a minimum separation of $\Lambda$, dashes represent additional $\Lambda / 2$ lengths. (c) Composite SEM image of the ADFB grating milled into a THz QCL ridge. Scale bar $200 \mu \mathrm{m}$. (d) Short section of an ADFB grating. Scale bar $50 \mu \mathrm{m}$. (e) Highmagnification image of a milled slit. Scale bar $500 \mathrm{~nm}$. (f) Cross-section through a milled slit of similar depth (though wider) in a second device. Scale bar $1 \mu \mathrm{m}$. (g) Dimensions of markers (cross and squares) milled alongside the QCL ridge with two overlapping ADFB sections (solid and dashed lines) (h) Typical marker alignment after ADFB milling, with sub- $\mu$ m stitching errors in $\mathrm{x}$ - and $\mathrm{y}$-directions. Scale bar $5 \mu \mathrm{m}$. 
Figure 3. THz QCL emission spectra before (dashed) and after (solid) introduction of ADFB gratings, along with the frequency placement of the grating reflectivity responses (lower panels). Vertical dotted lines indicate the centre frequency $\left(f_{\mathrm{N}}\right)$ of the grating responses. 
Figure 4. (a) Electrical and output power characteristics of three QCLs before and after ADFB introduction. (b) Top: thickness of the upper SI-SP waveguide layers. Below: representative SEM images of various ADFB device slits (scale bars $500 \mathrm{~nm}$ ), along with illustrations of their estimated cross-sectional milling profiles. (c) Number of lasing modes (above a $10 \mathrm{~dB}$ cut-off on a normalised power scale) versus estimated milling depth. (d) Average SMSR of the dominant post-FIB lasing modes. (e) Additional losses introduced to various QCLs by FIB-milled ADFB gratings, calculated from the elevated threshold driving current densities. Trend lines (dashed) in (c)-(e) have the same exponent $(d=z-235, \delta=110)$, revealing similar non-linearities in depth dependence of the presented device parameters. 
Figure 5. (a) Emission spectra from device $G$, before and after ADFB introduction. (b) Emission spectra from device $H$, before and after ADFB introduction. Vertical dotted lines indicate the centre frequency $\left(f_{\mathrm{N}}\right)$ of the grating responses. Lower panels: ADFB reflectivity responses. 


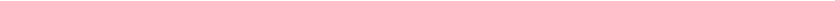




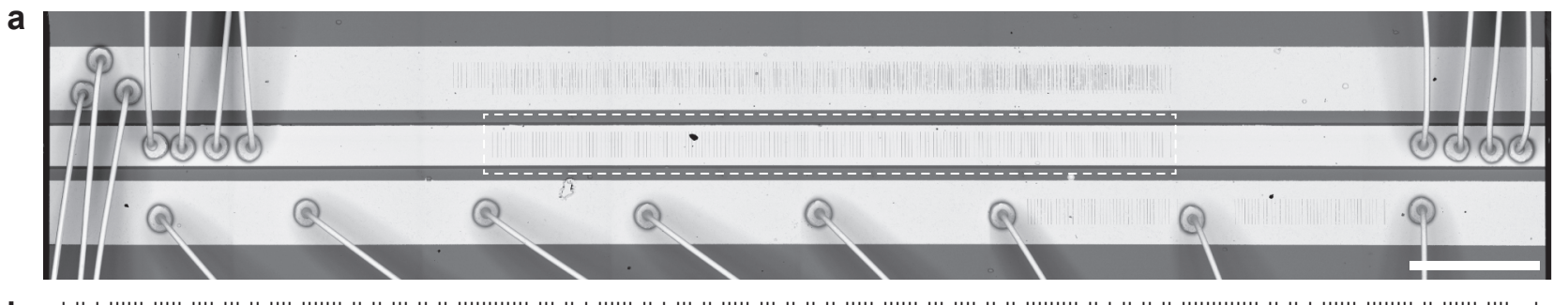

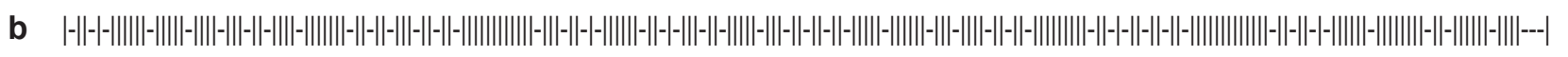
c

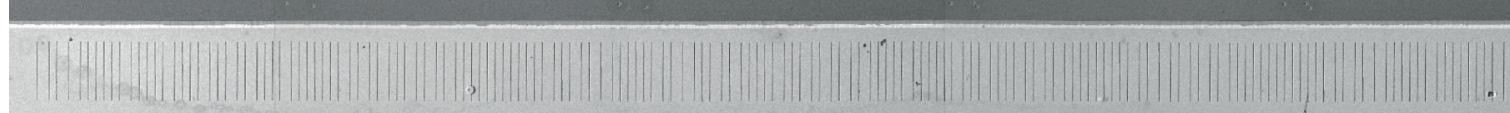

d

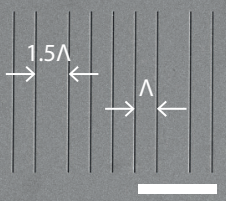

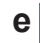

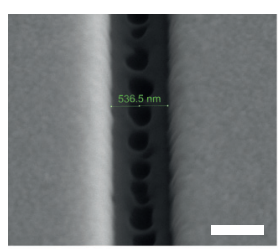

f

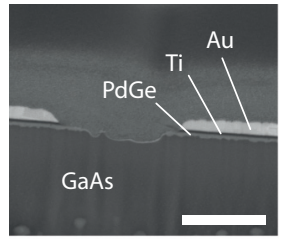

g

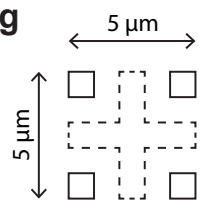

h
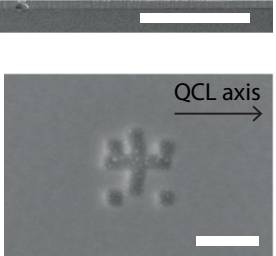


\section{Normalized frequency $\left(f / f_{N}\right)$}
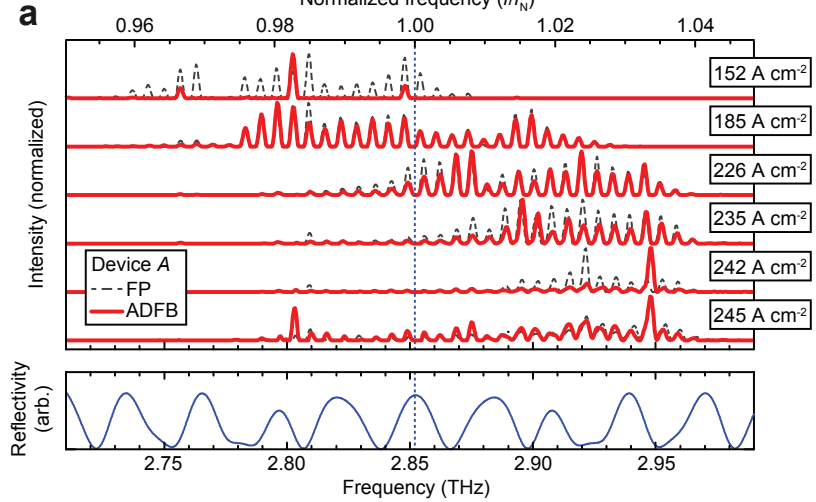

Normalized frequency $\left(f / f_{N}\right)$
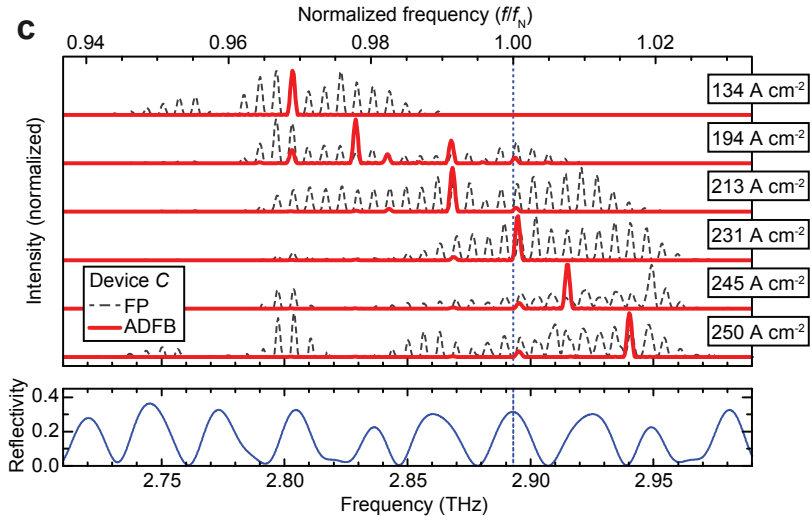

Normalized frequency $\left(f / f_{\mathrm{N}}\right)$

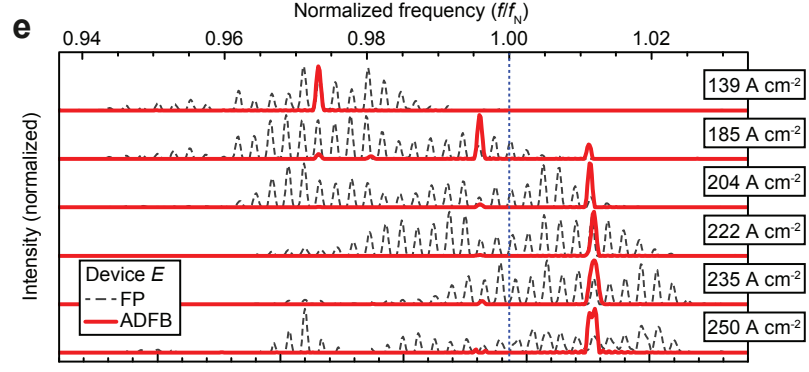

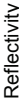

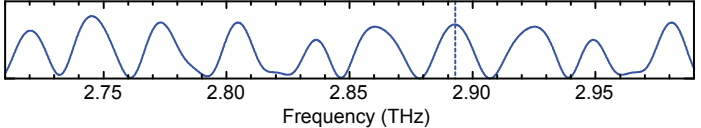

b

Normalized frequency $\left(f / f_{N}\right)$
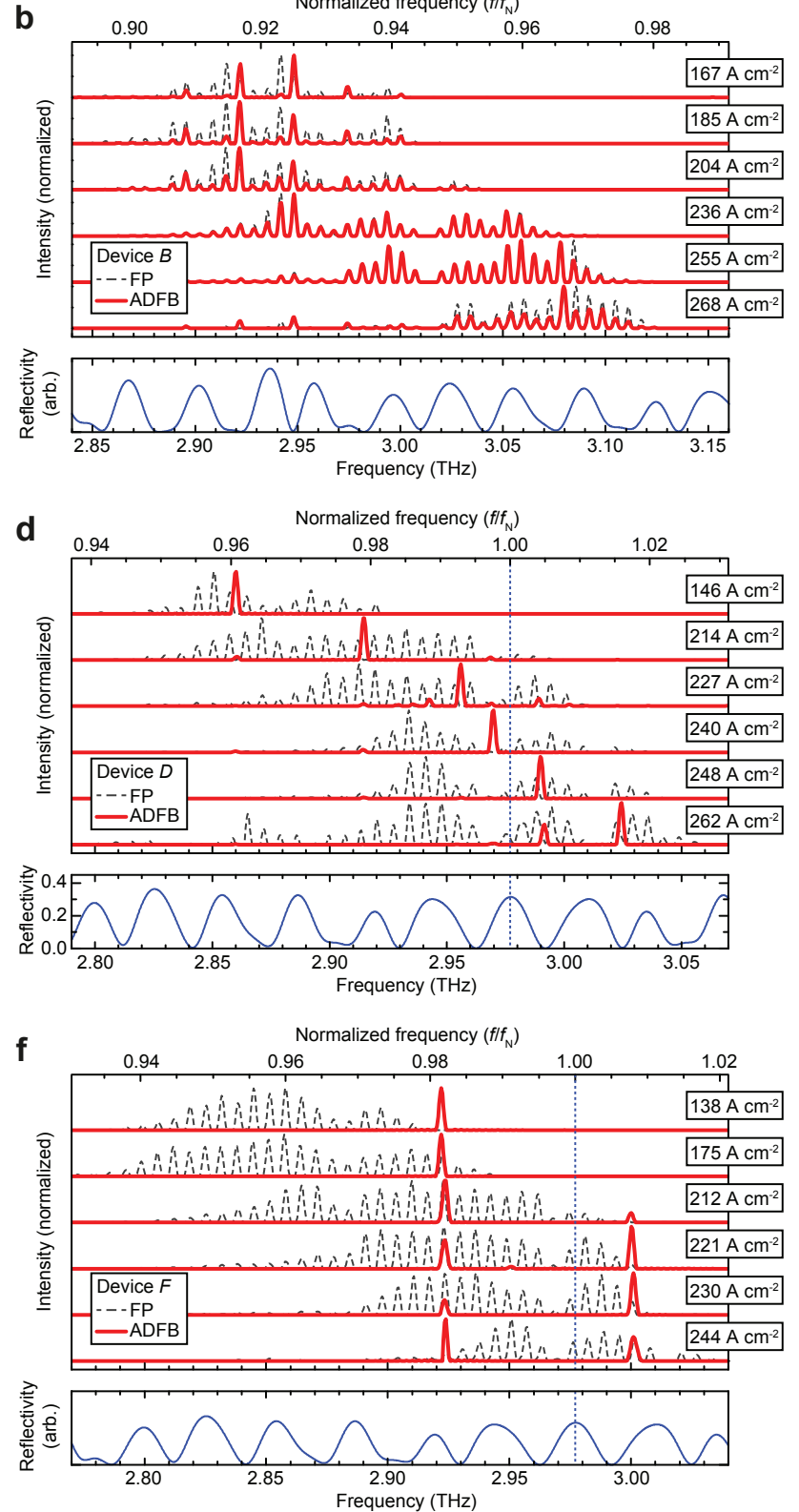


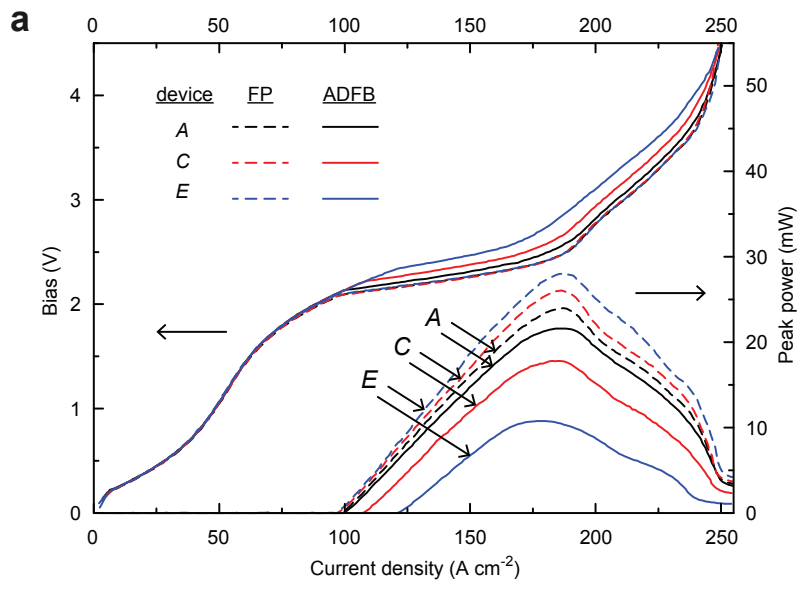

C $\quad \mathrm{Au} \quad \mathrm{Ti}$ PdGe $\mathrm{n}+\mathrm{GaAs} \quad$ Active region (AR)
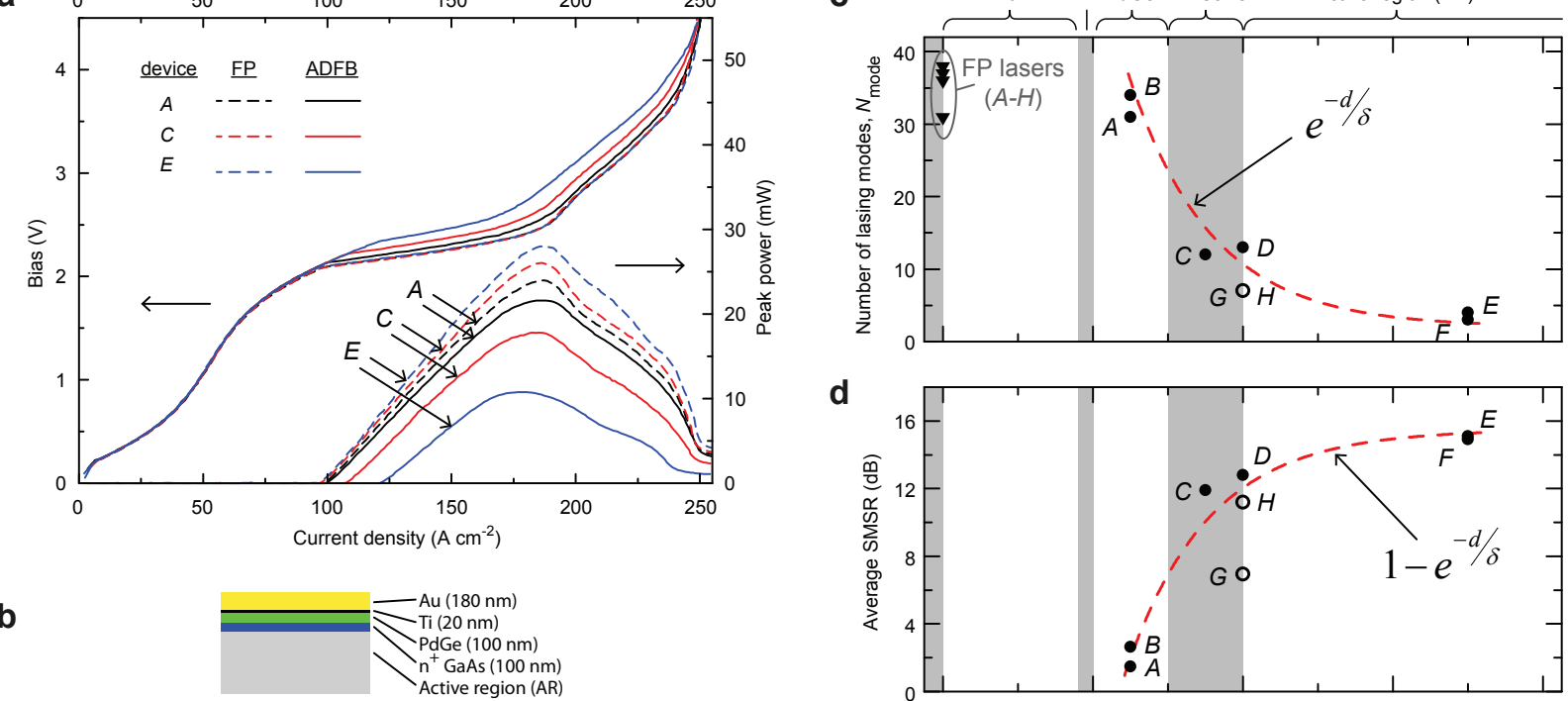

d

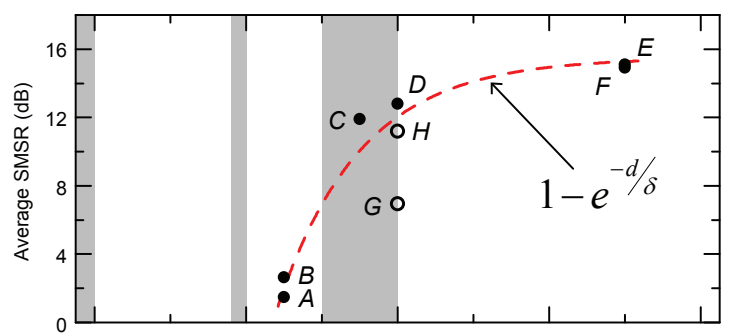

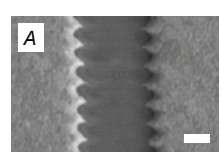
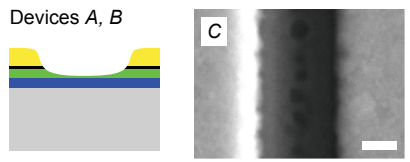

Device $C$

D

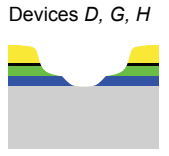

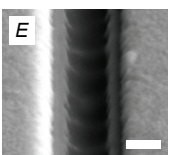

Devices $E, F$

- 不 $z$ e

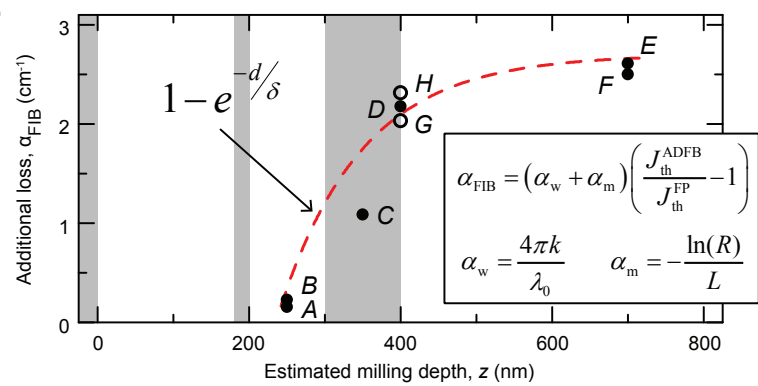




\section{Normalized frequency $\left(f / f_{N}\right)$}

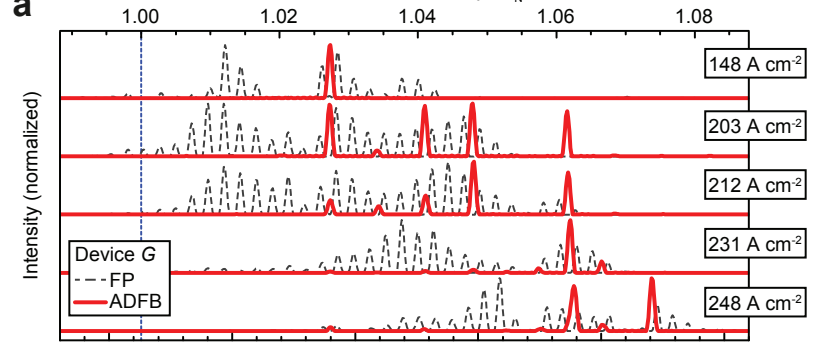

b

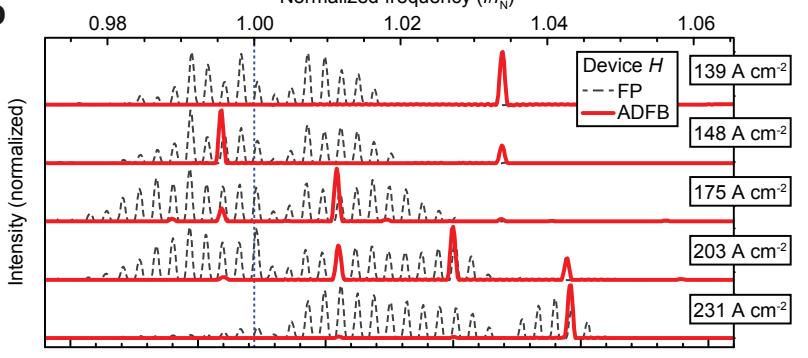

0.98 1.00

Normalized frequency $\left(f / f_{N}\right)$
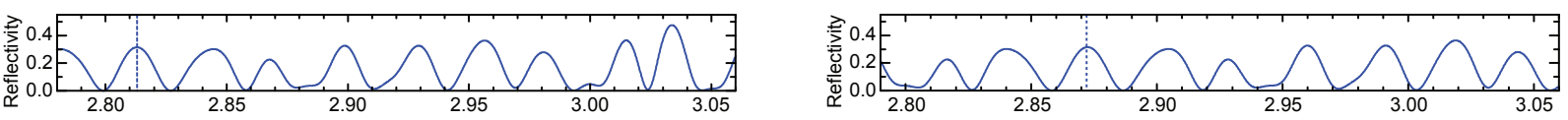

Frequency $(\mathrm{THz})$

Frequency $(\mathrm{THz})$ 